\title{
微生物对分解底物碳氮磷化学计量的响应和调节 机制
}

\section{周正虎 王传宽 ${ }^{*}$}

东北林业大学生态研究中心, 哈尔滨 150040

摘 要 微生物分解者的生存和生长策略、群落结构和功能会随着底物化学计量特征而改变, 从而强烈地影响底物的分解速 度和元素的周转速率, 进而影响生态系统的功能过程。深入理解微生物生理代谢活动和群落结构与陆地生态系统过程之间的 联系及其对全球变化的响应和反馈机理是生态学和全球变化生物学领域值得关注的重大科学命题。该文基于生态化学计量学 理论和代谢理论, 首先介绍微生物在陆地生态系统碳氮磷循环中的作用; 然后综述微生物对分解底物化学计量变异性的响应 和调节的4种主要机制: (1)调整微生物自身化学计量特征; (2)调整微生物群落结构; (3)产生特定的胞外酶以获取受限制的资 源; (4)改变碳氮磷元素利用效率。最后, 通过分析当前研究不足, 提出该领域亟需关注的科学问题有: (1)综合阐明微生物对底 物化学计量变化响应的各种机制及其相对重要性; (2)探索全球变化对微生物驱动的碳氮磷循环的影响; (3)探索微生物对底物 化学计量变化适应对策的时空变化。

关键词 生态化学计量; 土壤微生物; 酶; 生物地球化学循环; 内稳性; 碳利用效率; 养分利用效率

引用格式: 周正虎, 王传宽 (2016). 微生物对分解底物碳氮磷化学计量的响应和调节机制. 植物生态学报, 40, 620-630. doi: 10.17521/cjpe.2015.0449

\section{Responses and regulation mechanisms of microbial decomposers to substrate carbon, nitro- gen, and phosphorus stoichiometry}

ZHOU Zheng-Hu and WANG Chuan-Kuan*

Center for Ecological Research, Northeast Forestry University, Harbin 150040, China

\section{Abstract}

The survival and growth strategies, community structure and functions of microbial decomposers vary with substrate stoichiometry, which profoundly influences substrate decomposition, turnover, and hence the carbon and nutrient cycles of terrestrial ecosystems. It is crucial to understand the relationships among microbial metabolism, community structure and ecosystem processes of terrestrial ecosystems and their responses and feedbacks to global changes. In this review, we first introduced the significance of microbial decomposers in the carbon, nitrogen, and phosphorus cycles of terrestrial ecosystems from perspectives of ecological stoichiometry and metabolic theories. Then we synthesized four potential mechanisms of microbial response and control on substrate stoichiometric variations, i.e., through (1) modifying microbial stoichiometry, (2) shifting microbial community structure, (3) producing extracellular enzymes to acquire limiting resources, and (4) changing microbial carbon, nitrogen, and phosphor use efficiencies. Finally, we proposed three research directions in this field: (1) to comprehensively explore various microbial mechanisms in response to changes in substrate stoichiometry and the relative importance of these mechanisms; (2) to examine influences of global changes on microbial-driven cycles of carbon, nitrogen, and phosphorus; and (3) to explore spatiotemporal changes in the strategies of microbial adaptation to changes in the substrate stoichiometry.

Key words ecological stoichiometry; soil microbes; enzymes; biogeochemical cycle; homeostasis; carbon use efficiency; nutrient use efficiency

Citation: Zhou ZH, Wang CK (2016). Responses and regulation mechanisms of microbial decomposers to substrate carbon, nitrogen, and phosphorus stoichiometry. Chinese Journal of Plant Ecology, 40, 620-630. doi: 10.17521/cjpe.2015.0449

收稿日期Received: 2015-12-07 接受日期Accepted: 2016-03-26

* 通信作者Author for correspondence (E-mail: wangck-cf@nefu.edu.cn) 
微生物作为陆地生态系统主要分解者, 在获取 资源构建自身生物量的同时, 驱动着生态系统物质 和能量的流通, 调控着碳和养分在土壤-植物-大气 连续体(SPAC)之间的循环, 进而影响生态系统的结 构与功能(Chapin et al., 2003; Leff et al., 2015)。尽管 土壤微生物生物量碳只占土壤有机碳库的 $1.92 \%$ (Zhou \& Wang, 2015), 但其分解作用消耗了生物圈 的绝大部分净初级生产力(Cebrian \& Lartigue, 2004; 王兴昌和王传宽, 2015)。微生物代谢释放的 $\mathrm{CO}_{2}$ 占 土壤呼吸总通量的一半以上(Wang \& Yang, 2007), 其矿化和固氮作用维持了土壤的肥力(van der Heijen et al., 2008; Leff et al., 2015); 同时, 土壤微生物 与植物的共生、寄生和养分需求关系也会影响地上 植被生产力和生物多样性。因此, 探索陆地生态系 统地上植被组成和功能特征与地下微生物的耦合关 系成为目前生态学的研究热点(van der Heijen et al., 2008; Barberán et al., 2015; Moore et al., 2015); 认识 土壤微生物对碳氮磷循环的调控机制也是全球变化 背景下生物地球化学循环研究的重要内容 (Sinsabaugh \& Follstad Shah, 2011; Mooshammer et al., 2014; Zechmeister-Boltenstern et al., 2015)。

随着微生物群落鉴定技术的突破和完善、分子 生物学和同位素示踪在土壤微生物研究中的应用, 以土壤微生物为核心的生态系统变化过程与机理研 究正蓬勃发展。由于土壤微生物个体微小且数量庞 大, 其系统发生、生物多样性及其与生物和非生物 因素的相互作用远比动物和植物复杂, 因而微生物 生态学研究面临着巨大挑战(Prosser et al., 2007; Jansson \& Prosser, 2013)。运用生态化学计量学理论 (Sterner \& Elser, 2002)和代谢理论(Brown et al., 2004)研究土壤微生物, 不但可以提升我们对土壤 微生物生态过程和机理的认识, 而且有助于生态学 理论的发展与完善(Sterner \& Elser, 2002; Brown et al., 2004; Sinsabaugh \& Follstad Shah, 2011; Manzoni et al., 2012; Mooshammer et al., 2014; Buchkowski et al., 2015; Zechmeister-Boltenstern et al., 2015)。生 态化学计量学是研究生物系统能量平衡和多重化学 元素(主要是碳、氮、磷)平衡的科学(Sterner \& Elser, 2002); 代谢是指有机体从环境中获取能量和物质 资源, 以最优方式分配在存活、生长和繁殖上, 并将 废物排泄回环境(Brown et al., 2004)。这两者都结合 了生物学、化学、物理学等基本原理, 可以跨越生
物科学不同层次 $($ 从细胞新陈代谢到生态系统结构 和养分循环)探索不同生态系统组分之间的联系与 内在机理(Sterner \& Elser, 2002; Brown et al., 2004; Elser, 2006)。

微生物生态学研究的最终目标是建立微生物群 落结构及其代谢和生理活动与生态系统过程与机理 之间的联系(Prosser et al., 2007; Jansson \& Prosser, 2013)。我国对土壤微生物研究相对滞后, 在过去十 几年间, 主要关注于微生物生物量和群落结构与土 壤肥力或健康状况、温室气体排放、地上植被组成 等与环境因子之间的相关性, 以及对气候变暖、土 地利用变化、氮沉降等的响应, 而在理论和机理上 的研究尚不完善。为此, 本文基于生态化学计量学 和代谢理论, 首先介绍微生物在陆地生态系统碳氮 磷循环中的作用; 然后综述微生物对分解底物化学 计量变异性的主要响应和调节机制; 最后通过分析 当前研究不足, 提出该领域亟需关注的科学问题, 以期推动该领域的发展。

\section{1 微生物分解者与陆地生态系统碳氮磷循环}

土壤呼吸是陆地生态系统向大气圈排放的最大 碳源, 其中以土壤微生物代谢和生长释放出的 $\mathrm{CO}_{2}$ 为主的异养呼吸占总土壤呼吸一半以上(Högberg et al., 2001; Bond-Lamberty et al., 2004; Wang \& Yang, 2007), 因而在全球变化和生态系统研究中备受关 注(Wang et al., 2006)。然而, 以往研究多关注土壤呼 吸组分的划分以及与土壤温湿度、有机碳储量、植 被覆盖、土壤微生物生物量、环境因子等之间的相 关性(Högberg et al., 2001; Bond-Lamberty et al., 2004; Wang \& Yang, 2007; Wang et al., 2013), 但可 能由于对微生物生理代谢机制认识不足(Allison et al., 2010; Buchkowski et al., 2015; Steinauer et al., 2015), 未能阐明异养呼吸对复杂环境因子的响应 机制(Bardgett et al., 2008)。微生物驱动的异养呼吸 与微生物生长所需能量投资密切相关, 关系到微生 物在呼吸代谢和生物量生长之间的碳资源分配。因 此, 微生物将底物碳转化为自身生物量碳的效率 (即微生物碳利用效率, CUE; del Giorgio \& Cole, 1998)与陆地生态系统碳循环直接相关(Manzoni et al., 2012; Mooshammer et al., 2014; Buchkowski et al., 2015; Zechmeister-Boltenstern et al., 2015)。

氮和磷是陆地生态系统生产力的关键限制元素 
(Vitousek \& Howarth, 1991; Vitousek et al., 2010)。微 生物分解者主要通过驱动如下过程来调节陆地生态 系统氮循环：通过共生固氮、自生固氮、联合固氮 等将大气中分子氮还原为铵态氮; 优先吸收铵态氮 后同化为有机氮; 通过胞外酶将资源中有机氮转化 为无机氮的过程(即矿化作用); 通过自养硝化细菌 的硝化作用将氨氧化为硝态氮; 通过反硝化细菌的 反硝化作用将硝态氮还原为氮气或氧化亚氮重新进 入大气圈(Jetten, 2008; Rick \& Thomas, 2001)。尽管 微生物不能像固氮作用一样增加土壤磷总含量, 但 土壤微生物的活动会显著改变土壤中磷的化学形式 和比例, 从而在调节植物磷可利用性中扮演重要角 色(van der Heijden et al., 2008)。例如: 微生物分解 有机质释放有效磷; 在磷限制的生态系统中, 微生 物生物量磷占土壤总磷的比例可达到 $23.6 \%$ (天然 湿地生态系统, Xu et al., 2013); 又因为土壤微生物 生物量的最大值出现在冬季, 使得在植被生长季中 土壤微生物生物量减小时释放出磷, 及时增加土壤 中磷的可利用性(Richardson \& Simpson, 2011; Xu et al., 2013)。另外, 土壤微生物的存在还会改变土壤 的吸附平衡, 从而增加正磷酸盐向土壤溶液中的转 移(Seeling \& Zasoski, 1993)。由此可见, 微生物分解 者在陆地生态系统生物地球化学循环中起着重要的 驱动和调节作用。

\section{2 微生物分解者对底物元素化学计量的响 应和调节}

地球上所有生物的生长和繁殖都需要 25 种相同 的化学元素, 但这些元素的相对比例(及化学计量) 在生物体之间存在巨大差异; 这一方面取决于生物 有机体自身的结构限制和代谢需求(Sterner \& Elser, 2002; Brown et al., 2004), 另一方面也受环境影响 而表现出一定环境可塑性(Sardans et al., 2012; Yang et al., 2015)。

陆地生态系统中微生物的分解底物主要来源于 植物的枯枝落叶、粗木质残体、根系调落物和分泌 物、土壤有机质等 (Mooshammer et al., 2014; Buchkowski et al., 2015; Zechmeister-Boltenstern et al., 2015)。在全球尺度上, 森林生态系统中植物叶 片 C:N:P为 1 212:28:1 (摩尔比, McGroddy et al., 2004)。植物叶片在调亡过程中的养分再吸收作用, 使得枯叶养分含量骤减, 从而使枯叶的 C:N:P变为
3 007:45:1 (McGroddy et al., 2004; Yuan \& Chen, 2009; Reed et al., 2012; Vergutz et al., 2012; Han et al., 2013)。此外, 枯叶的 C:N:P还会随生态系统、生 物区系以及生活型的不同而发生显著变化 (McGroddy et al., 2004)。粗木质残体和枯立木的现 存量占生态系统碎屑碳库的45\% (Zhang \& Wang, 2010); 粗木质残体占整个生态系统植物碎屑年输 入量的10\% (温带森林, Harmon et al., 1986)至30\% (热带森林, Martinelli et al., 2000), 其C:N:P可高达 14 103:40:1 (Weedon et al., 2009)。植物细根周转产 生的调落物是土壤碳和养分的重要来源, 其死根 $\mathrm{C}: \mathrm{N}: \mathrm{P}$ 的全球平均值为 4 184:43:1 (Yuan et al., 2011)。土壤C:N:P最低(287:17:1, Xu et al., 2013), 且 随着土壤深度增加而显著减小(Tian et al., 2010)。相 比之下, 土壤微生物生物量 C:N:P比其分解底物低 得多。例如: Cleveland和Liptzin (2007)整合全球数据 时发现, 陆地生态系统土壤微生物生物量碳氮磷化 学计量虽然存在类似于海洋浮游植物的“Redfield ratio” (在资源不受限制时浮游植物的 C:N:P为 106:16:1; Redfield, 1958), 即土壤微生物生物量 $\mathrm{C}: \mathrm{N}: \mathrm{P}$ 具有内稳性 (homeostasis; Sterner \& Elser, 2002), 但森林和草地的土壤微生物生物量 $\mathrm{C}: \mathrm{N}: \mathrm{P}$ 之 间存在显著差异。近期Xu等(2013)进一步整合分析 表明, 土壤微生物生物量 $\mathrm{C}: \mathrm{N}: \mathrm{P}$ 随生物区系不同有 很大的变异性 (从沙漠的 31:4:1 到天然湿地的 131:14:1)。面临底物和微生物化学计量的巨大变异 性, 微生物主要通过调整自身化学计量特征、群落 结构、胞外酶比例、元素利用效率等4种机制来响应 和调节(图1)。

\section{1 微生物化学计量的可塑性}

生长速率理论(growth rate hypothesis)指出: 高 生长速率需要增加对富含磷元素的核糖体RNA的 投资, 意味着其 $\mathrm{N}: \mathrm{P}$ 值的减小(Elser et al., 2003)。 Li 等(2014)发现，土壤微生物生物量 $\mathrm{N}: \mathrm{P}$ 与植物叶片、 枯叶以及细根的 $\mathrm{N}: \mathrm{P}$ 均随着纬度、年平均气温、年 降水量而呈现出完全一致的变化趋势, 即低纬度地 区比高纬度地区更受磷素限制。这种 $\mathrm{N}: \mathrm{P}$ 格局不但 支持生长速率理论, 而且也表明微生物和植物一样 在高纬度地区由于生长季短而需要快速的生长速 率, 进而需要高的氮、磷含量和低的 $\mathrm{N}: \mathrm{P}$ 支持(Elser et al., 2003; Makino et al., 2003; Lovelock et al., 2007; Borer et al., 2013; Zechmeister-Boltenstern 


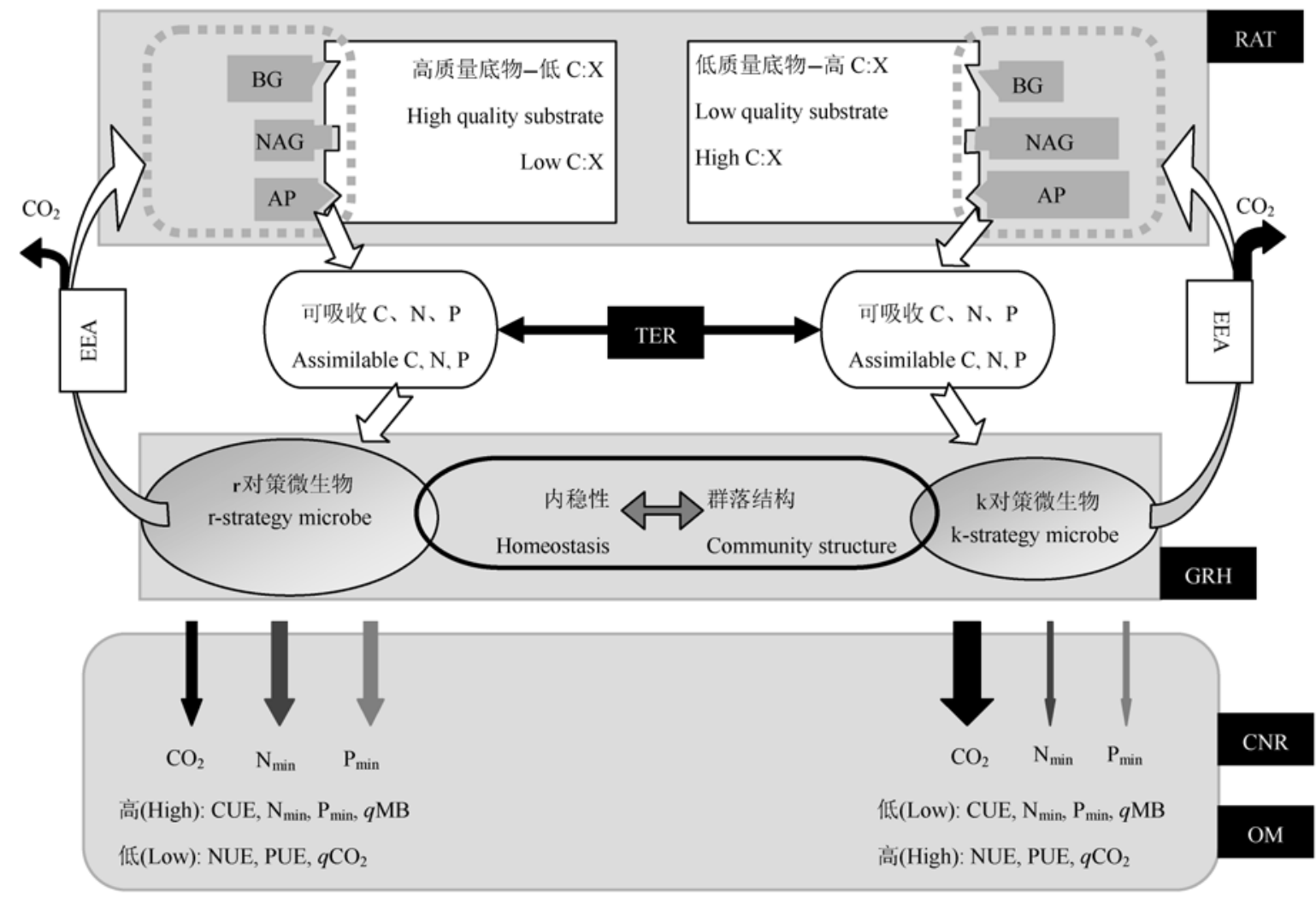

图1 微生物分解者对底物化学计量的响应和调节机制。C:X, 底物碳和养分(氮和磷)比; BG, $\beta-1,4-$ 葡萄糖苷酶; NAG, $\beta-1,4-\mathrm{N}-$ 乙酰葡糖氨糖苷酶; AP, 磷酸酶; EEA，胞外酶活性; RAT, 资源分配理论; TER, 元素比率阈值; GRH, 生长速率理论; CNR, 消 费者驱动的养分循环; OM, 溢出代谢; CUE, 碳利用效率; NUE, 氮利用效率; PUE, 磷利用效率; $q \mathrm{MB}$, 微生物生物量碳在底 物总有机碳中的比例; $q \mathrm{CO}_{2}$, 单位微生物生物量碳呼吸速率; $\mathrm{N}_{\min }$, 氮矿化; $\mathrm{P}_{\min }$ 磷矿化。虚线方框中多边形大小表示胞外酶活 大小; 椭圆大小表示微生物生物量大小; 箭头的宽窄表示碳氮磷通量大小。

Fig. 1 Mechanisms of microbial response and regulation on substrate stoichiometry. C:X, substrate carbon to nutrient (nitrogen and phosphor) ratios; BG, $\beta$-1,4-glucosidase; NAG, $\beta$-1,4-N-acetylglucosaminidase; AP, phosphatase, EEA, extracellular enzymatic activity; RAT, resource allocation theory; TER, threshold elemental ratio; GRH, growth rate hypothesis; CNR, consumer-driven nutrient recycling; OM, overflow metabolism; CUE, carbon use efficiency; NUE, nitrogen use efficiency; PUE, phosphor use efficiency; $q \mathrm{MB}$, fraction of total substrate carbon in microbe; $q \mathrm{CO}_{2}$, microbial respiration rate per unit biomass carbon; $\mathrm{N}_{\text {min }}$, nitrogen mineralization; $\mathrm{P}_{\min }$, phosphor mineralization. Size of the polygons in the dotted box indicates the strength of enzymatic activity; size of the ellipses indicates the amount of microbial biomass; and the width of the arrows indicates the magnitude of the carbon, nitrogen, and phosphor fluxes.

et al., 2015)。此外, 尽管以往研究普遍认为微生物 生物量C:N变异性很小(Makino et al., 2003), 然而 Zhou和Wang (2015)最近整合中国森林土壤微生物 生物量碳氮格局时发现，土壤资源(土壤有机碳、全 氮)和气候条件(年平均气温、年降水量)共同解释了 土壤微生物生物量C:N的 $24.4 \%$ 的变异性, 其中土壤 资源和气候条件各占一半。由此可见, 针对底物化 学计量的变异性, 微生物的适应对策之一是其个体 生物量C:N:P也随之变化, 即非内稳性(non-homeostasis), 这被Sterner和Elser (2002)形象地描述为“You are what you eat”。然而, 微生物生物量 $\mathrm{C}: \mathrm{N}: \mathrm{P}$ 与底物 $\mathrm{C}: \mathrm{N}: \mathrm{P}$ 之间的关系仍没有定论。例如: Li等(2012)分
析我国亚热带地区1069个土壤数据时报道, 微生 物生物量 $\mathrm{C}: \mathrm{N}$ 和 $\mathrm{C}: \mathrm{P}$ 随着土壤 $\mathrm{C}: \mathrm{N}$ 和 C:P的增加而显 著增加, 微生物生物量 $\mathrm{N}: \mathrm{P}$ 却随着土壤 $\mathrm{N}: \mathrm{P}$ 的增加而 呈现减小的趋势。Zhou和Wang (2015)发现我国森林 土壤微生物生物量 $\mathrm{C}: \mathrm{N}$ 随着土壤 $\mathrm{C}: \mathrm{N}$ 的增加反而减 小, 与Cleveland和Liptzin (2007)的全球森林数据分 析结果吻合。而Fanin等(2013)的调落物分解实验却 指出微生物生物量 $\mathrm{C}: \mathrm{P}$ 和 $\mathrm{N}: \mathrm{P}$ 均与调落物浸出液 $\mathrm{C}: \mathrm{P}$ 和 $\mathrm{N}: \mathrm{P}$ 显著正相关。产生这种现象的可能原因是微 生物化学计量比会随着研究区域气候条件的变化而 变化; 大尺度上的全球整合分析夹杂了气候、土壤 类型等因素的影响, 而小尺度或个体研究也许更能 
反映微生物化学计量比与底物化学计量比的相关 性。因此, 微生物与底物化学计量之间关系的方向 与强度还需要进一步验证。

\section{2 微生物群落结构的可塑性}

在微生物群落水平上, 自然选择会使那些能够 充分获取并利用特定资源的种群在竞争中获胜 (Moorhead \& Sinsabaugh, 2006; Fierer et al., 2007; Strickland \& Rousk, 2010; Martin et al., 2015; Zelezniak et al., 2015)。因此, 底物的质量(高质量意味着 低的碳与养分比(C:X), 低质量意味着高的C:X; Xu et al., 2014)的改变必然会改变微生物群落组成。目 前很多学者认为, 微生物生物量 C:N:P的可塑性是 由微生物群落结构的变化导致的(Fanin et al., 2013; Kaiser et al., 2014; Mooshammer et al., 2014; Zechmeister-Boltenstern et al., 2015)。生长和周转迅速的r 对策微生物常具有较低的生物量 $\mathrm{C}: \mathrm{X}$, 适应于高质 量的底物; 相反, 生长和周转速度较慢的 $\mathrm{k}$ 对策微 生物常具有较高的生物量 C:X, 适应于低质量底物 (Moorhead \& Sinsabaugh, 2006; Fierer et al., 2007; Strickland \& Rousk, 2010; Kaiser et al., 2014)。然而, 由于微生物数量、群落组成以及代谢活动的复杂性, 微生物群落结构、生物量 $\mathrm{C}: \mathrm{N}: \mathrm{P}$ 与底物 $\mathrm{C}: \mathrm{N}: \mathrm{P}$ 之间的 关系仍不明确。例如：由于真菌的 C: $\mathrm{N}$ 比细菌高 (Strickland \& Rousk, 2010), 且真菌与细菌的比例以 及底物 C: $\mathrm{N}$ 均随着土壤剖面而降低(Moore et al., 2010; Lee et al., 2013)。Mooshammer等(2014)在分析 微生物分解者对底物质量的适应机制时推测, 真菌 与细菌比例应该随着底物 C: $\mathrm{N}$ 的增加而增加。Fanin 等(2013)的确发现微生物生物量C: $\mathrm{N}$ 与真菌与细菌比 例存在显著的相关性, 但真菌与细菌比例与底物 $\mathrm{C}: \mathrm{N}: \mathrm{P}$ 并无相关性。然而, 同属于子囊菌门(Ascomycota) 的刺盾贫纲(Chaetothyriomycetes)的真菌适应 于低质量底物, 而锤舌菌纲(Leotiomycetes)和粪壳菌 纲(Sordariomycetes)的真菌却适应于高质量的底物; 这表明, 即便是在真菌内部, 不同分类单元对底物 质量的要求也不尽相同(Strickland et al., 2009; Schneider et al., 2012)。因此, 微生物群落结构、生物量 $\mathrm{C}: \mathrm{N}: \mathrm{P}$ 与底物 $\mathrm{C}: \mathrm{N}: \mathrm{P}$ 之间普适性的关系还需验证。

\section{3 微生物胞外酶化学计量的调整}

微生物群落产生的胞外酶 (extracellular enzymatic activities, EEA)在体外将植物残体分解为活性 组分, 以利于自身吸收与同化(Sinsabaugh et al.,
2008)。通常认为, 用于获取碳、氮、磷的主要胞外 酶分别为 $\beta-1,4-$ 葡萄糖苷酶(BG)、 $\beta-1,4-\mathrm{N}-$ 乙酰葡糖 氨糖苷酶(NAG)、酸生/碱性磷酸酶(AP); 这3种胞外 酶的比例关系(BG:NAG:AP)被称为“生态酶化学计 量”(ecoenzymatic stoichiometry), 它可以反映微生 物生物量 $\mathrm{C}: \mathrm{N}: \mathrm{P}$ 与底物 $\mathrm{C}: \mathrm{N}: \mathrm{P}$ 以及与微生物的同化和 生长效率之间的联系(Sinsabaugh et al., 2008, 2009; Sinsabaugh \& Follstad Shah, 2011, 2012; Waring et al., 2014)。资源分配理论(resource allocation theory; Allison \& Vitousek, 2005)指出微生物在受单一元素 限制时会增加与该元素对应的胞外酶的合成。然而, 某种胞外酶的增加需要能量和物质的投资。其中, 能量的消耗需要更多的碳供应, 意味着高的呼吸速 率; 而物质的投资主要需要更多的碳和氮供应，而 且胞外酶C:N (平均值为3)显著低于微生物生物量 $C: N$ 。因此, 资源分配理论具有一定的局限性 (Schimel \& Weintraub, 2003)。一般而言, 资源分配 理论能较好地解释微生物受到磷限制时的机理。例 如: 磷酸酶通常与资源有效磷成反比(即磷添加会 抑制磷酸酶); 而氮添加会促使微生物产生更多的 磷酸酶(Marklein \& Houlton, 2012)。全球尺度上, 受 磷限制的热带地区的BG:AP和NAG:AP也显著低于 温带地区, 并且风化程度高的土壤具有低的BG:AP 和NAG:AP (Waring et al., 2014)。然而, 磷酸酶的这 些响应的程度还取决于土壤中碳氮的可利用性 (Schimel \& Weintraub, 2003)。当受到氮限制时, 微 生物通常不会消耗自身氮素而产生更多的与氮获取 相关的胞外酶; 当受到严重氮限制时, 微生物很可 能在氮限制解除之前一直处于休眠状态(Sinsabaugh \& Follstad Shah, 2012; Blagodatskaya \& Kuzyakov, 2013)。微生物对碳和氮的获取相协调, 主要通过吸 收氨基酸和氨基糖而获取; 而微生物对磷的获取是 先将以酯键相连的有机磷通过磷酸酶水解成无机磷 而获取。这就导致BG:AP和NAG:AP具有环境和底 物变异性, 而 BG:NAG更加保守 (Waring et al., 2014)。因此, 微生物通过胞外酶的化学计量调整来 适应底物化学计量变异性的机理还需要进一步地研 究和证实。

\section{4 微生物资源利用效率的调整}

消费者驱动的养分循环理论指出, 资源 $\mathrm{C}: \mathrm{N}: \mathrm{P}$ 和消费者 $\mathrm{C}: \mathrm{N}: \mathrm{P}$ 以及消费者的元素利用效率之间的 平衡直接影响到生态系统碳氮磷的流通(Sterner \& 
Elser, 2002)。微生物会保留自身组成元素维持和代 谢需求的限制性元素, 排出多余的元素, 这一过程 被称为微生物消费者驱动的养分循环 (consumerdriven nutrient recycling; Sterner \& Elser, 2002)。例 如: 当微生物生长在低质量底物环境中时, 微生物 会通过呼吸释放出过量的碳来满足自身养分需求的 代谢活动, 这一现象被称为溢出代谢或溢出呼吸 (overflow metabolism/respiration; Hessen et al., 2013; Schimel \& Weintraub, 2003; Sinsabaugh et al., 2013)。 最新研究表明, 单位微生物生物量碳呼吸速率会随 着底物 C:X的增加而显著增加, 一定程度上反映了 微生物的溢出呼吸(Spohn, 2015; Spohn \& Chodak, 2015)。但微生物呼吸的增加是否伴随着更多能量投 入到胞外酶的生产上, 尚需要进一步验证(Hartman \& Richardson, 2013; Spohn, 2015; Spohn \& Chodak, 2015)。相反。当微生物生长于高质量底物环境中时, 微生物氮磷矿化速率会随之增加; 这两者之间的相 关性已被广泛证实(Manzoni et al., 2010; Mooshammer et al., 2012; Deng et al., 2014; Buchkowski et al., 2015)。Zhou和Wang (2015)最近还发现, 微生物 生物量碳在土壤总有机碳中所占的比例随土壤C:N 的增加而显著减小, 但微生物生物量氮在土壤总氮 中所占的比例却随土壤 $\mathrm{C}: \mathrm{N}$ 的增加而显著增加, 这 表明底物 C:X还会影响微生物生物量大小以及土壤 的元素化学计量。

通常将生物对底物 $\mathrm{C}: \mathrm{X}$ 所能承受的限度定义为 元素比率阈值(threshold elemental ratio)。在微生物 研究中, 元素比率阈值建立在代谢理论和化学计量 理论基础上, 整合了微生物生物量 $\mathrm{C}: \mathrm{N}: \mathrm{P}$ 、胞外酶化 学计量关系(BG:NAG:AP), 以及元素利用效率对底 物 C:N:P的适应策略(Sinsabaugh \& Follstad Shah, 2012)。如果底物C: $X$ 超过这个阈值, 则微生物受养分 限制, 使其碳利用效率随底物C:X的增加而减小、养 分利用效率会增加(Mooshammer et al., 2014; Zechmeister-Boltenstern et al., 2015); 反之, 当底物C:X低 于这个阈值时, 微生物受到能量(碳)限制, 使其碳利 用效率随底物 $\mathrm{C}: \mathrm{X}$ 的增加而增加, 而养分利用效率会 降低(Sterner \& Elser, 2002; Frost et al. 2006; Doi et al. 2010; Sinsabaugh \& Follstad Shah, 2012)。

\section{3 研究展望}

微生物是陆地生态系统的主要分解者, 其生存
和生长策略、群落结构和功能会随着底物化学计量 特征而改变, 从而强烈地影响底物的分解速度和元 素的周转速率, 进而影响生态系统的功能过程 (Manzoni et al., 2010; Sinsabaugh \& Shah, 2011; Mooshammer et al., 2012; Xu et al., 2014; Buchkowski et al., 2015; Zechmeister-Boltenstern et al., 2015; Zhou \& Wang, 2015)。深入理解微生物生理代 谢活动和群落结构与陆地生态系统过程之间联系及 其对全球变化的响应和反馈机理, 是生态学和全球 变化生物学领域值得关注的重大科学命题, 其中亟 待解决的主要科学问题有:

(1)综合阐明微生物对底物化学计量变化响应 的各种机制及其相对重要性。尽管已经提出上述4 种微生物适应底物化学计量变化的可能机制, 但每 种机制还需要进一步验证，尤其需要通过综合性实 验研究在不同时空尺度上各种机理的相对贡献程 度。具体来说, 微生物生物量C:N:P的可塑性同时受 底物化学计量、气候、土壤理化性质等众多因子影 响, 因此微生物生物量 $\mathrm{C}: \mathrm{N}: \mathrm{P}$ 与底物 $\mathrm{C}: \mathrm{N}: \mathrm{P}$ 的关联性 及其强度有待于量化。虽然近期大量研究关注底物 $\mathrm{C}: \mathrm{N}: \mathrm{P}$ 与微生物群落的相关性(Fanin et al., 2013; Kaiser et al., 2014), 但仍然没有普适性的结论。理论 上, 微生物群落结构的变化必然导致微生物生物量 $\mathrm{C}: \mathrm{N}: \mathrm{P}$ 的变化, 但区分其贡献程度仍有难度。化学计 量学通过简单、统一而又本质的元素计量特征将复 杂的生态系统层次有机地联系起来, 考虑微生物群 落结构可能反而将问题复杂化。胞外酶在解释微生 物对氮的获取和利用上仍具有很大的局限性。对微 生物元素利用效率研究的不足主要体现在缺乏可靠 的测定方法。目前主要通过添加同位素标记的特定 基质进行测定，但微生物同时也会利用未标记的土 壤有机质, 因而测定结果具有不确定性(Sinsabaugh et al., 2013)。至于回答各种机理在什么条件下起主 导作用, 以及相对贡献程度等问题, 还需要设计综 合实验。

(2)探索全球变化对微生物驱动的碳氮磷循环 的影响。整合分析表明全球变暖、大气 $\mathrm{CO}_{2}$ 浓度升 高、干旱等均会导致植物 C:N和C:P的增加(Sardans \& Peñuelas, 2012), 底物C:X的增加会导致微生物呼 吸的增加, 从而影响土壤碳固存。与此同时, 微生物 生理代谢本身对全球变暖、大气 $\mathrm{CO}_{2}$ 浓度升高、干 旱等也很敏感。氮沉降的持续增加会降低植物 C: $N$, 
增加N:P (Sardans \& Peñuelas, 2012; 王常顺和汪诗 平, 2015), 提高微生物碳利用效率, 减弱微生物呼 吸作用, 从而促进土壤碳积累(李嵘和常瑞英, 2015)。然而, 氮沉降的增加也会导致年调落物量的 增加(Adamek et al., 2009; 李嵘和常瑞英, 2015), 会 诱导微生物生物量的增加, 转而可能会因受到磷限 制而影响微生物呼吸。最近研究还表明, 微生物C:N 会随着氮添加而减小(Fisk et al., 2015)。然而, 全球 变暖、大气 $\mathrm{CO}_{2}$ 浓度升高、干旱对微生物化学计量 比的直接影响还不明确。因此, 全球变化对微生物 和底物的直接和间接效应如何影响微生物对底物化 学计量的适应对策, 尚需探索。

(3)探索微生物对底物化学计量变化适应对策 的时空变化。Odum (1969)生态系统演替理论指出, 生态系统呼吸和生物量的比例会随着演替进程而减 小。Insam和Haselwandter (1989)首次提出生态系统 演替过程中微生物也有类似的变化趋势, 即单位微 生物生物量的呼吸速率随着演替进程而减小。但 Wardle和Ghani (1995)的整合研究发现大多数情况 下单位微生物生物量的呼吸速率随着演替进程先减 后增。此后相继出现大量研究, 但仍无统一结论和 解释(Saviozzi et al., 2001; Goberna et al., 2006; Susyan et al., 2011; Lu et al., 2014; Raiesi \& Beheshti, 2015)。运用化学计量学理论可能有助于解释这些争 议: 随着生态系统的演替进程, 一方面植物光合作 用和微生物固氮作用将大气中的碳和氮素不断输入 土壤, 从而导致土壤 $\mathrm{C}: \mathrm{P}$ 和 $\mathrm{N}: \mathrm{P}$ 的增加; 另一方面, 调落物、地表枯落物层、土壤 $\mathrm{C}: \mathrm{N}$ 随着演替进程基 本保持不变(Yang \& Luo, 2011; Yang et al., 2011; 周 正虎等, 2015), 而单位微生物生物量的呼吸速率随 着C:P的增加而增加(图1; Spohn \& Chodak, 2015), 从而出现Wardle和Ghani (1995)的“先减后增”格局。 另外, 普遍认为, 高纬度地区受氮限制, 而低纬度 地区受磷限制; 生态系统演替初期受氮限制, 而后 期受磷限制(Vitousek et al., 2010)。然而, Spohn和 Chodak (2015)在德国发现温带森林土壤C:P与单位 微生物生物量的呼吸速率显著相关, 而与 C:N不相 关; 长期监测结果也显示, 该地区森林植物叶片的 磷含量也在减小(Ilg et al., 2009)。因此, 上述生态系 统演替和全球变化(尤其是氮沉降)可能会导致生态 系统的磷限制而突破磷会受纬度限制的传统认识 (Vitousek et al., 2010)。
基金项目 国家“十二五”科技支撑项目 (2011BAD37B01)、教育部长江学者和创新团队发展计划 (IRT_15R09)。

\section{参考文献}

Adamek M, Corre MD, Hölscher D (2009). Early effect of elevated nitrogen input on above-ground net primary production of a lower montane rain forest, Panama. Journal of Tropical Ecology, 25, 637-647.

Allison SD, Vitousek PM (2005). Responses of extracellular enzymes to simple and complex nutrient inputs. Soil Biology \& Biochemistry, 37, 937-944.

Allison SD, Wallenstein MD, Bradford MA (2010). Soil-carbon response to warming dependent on microbial physiology. Nature Geoscience, 3, 336-340.

Barberán A, McGuire KL, Wolf JA, Jones FA, Wright SJ, Turner BL, Fierer N (2015). Relating belowground microbial composition to the taxonomic phylogenetic and functional trait distributions of trees in a tropical forest. Ecology Letters, 18, 1397-1405.

Bardgett RD, Freeman C, Ostle NJ (2008). Microbial contributions to climate change through carbon cycle feedbacks. The ISME Journal, 2, 805-814.

Blagodatskaya E, Kuzyakov Y (2013). Active microorganisms in soil: Critical review of estimation criteria and approaches. Soil Biology \& Biochemistry, 67,192-211.

Bond-Lamberty B, Wang CK, Gower ST (2004). A global relationship between the heterotrophic and autotrophic components of soil respiration. Global Change Biology, 10, 1756-1766.

Borer ET, Bracken MES, Seabloom EW, Smith JE, Cebrian J, Cleland EE, Elser JJ, Fagan WF, Gruner DS, Harpole WS, Hillebrand H, Kerkhoff AJ, Ngai JT (2013). Global biogeography of autotroph chemistry: Is insolation a driving force? Oikos, 122, 1121-1130.

Brown JH, Gillooly JF, Allen AP, Savage VM, West GB (2004). Toward a metabolic theory of ecology. Ecology, 85, 1771-1789.

Buchkowski RW, Schmitz OJ, Bradford MA (2015). Microbial stoichiometry overrides biomass as a regulator of soil carbon and nitrogen cycling. Ecology, 964, 1139-1149.

Cebrian J, Lartigue J (2004). Patterns of herbivory and decomposition in aquatic and terrestrial ecosystems. Ecological Monographs, 74, 237-259.

Chapin CT, Bridgham SD, Pastor J, Updegraff K (2003). Nitrogen phosphorus and carbon mineralization in response to nutrient and lime additions in peatlands. Soil Science, 168, 409-420.

Cleveland CC, Liptzin D (2007). C:N:P stoichiometry in soil: Is there a "Redfield ratio" for the microbial biomass? Biogeochemistry, 85, 235-252.

del Giorgio PA, Cole JJ (1998). Bacterial growth efficiency in natural aquatic systems. Annual Review of Ecology \&

www.plant-ecology.com 
Systematics, 29, 503-541.

Deng Q, Cheng XL, Yang YH, Zhang QF, Luo YQ (2014). Carbon-nitrogen interactions during afforestation in central China. Soil Biology \& Biochemistry, 69, 119-122.

Doi H, Cherif M, Iwabuchi T, Katano I, Stegen JC, Striebel M (2010). Integrating elements and energy through the metabolic dependencies of gross growth efficiency and the threshold elemental ratio. Oikos, 119, 752-765.

Elser JJ (2006). Biological stoichiometry: A chemical bridge between ecosystem ecology and evolutionary biology. The American Naturalist, 168, S25-S35.

Elser JJ, Acharya K, Kyle M, Cotner J, Makino W, Markow T, Watts T, Hobbie S, Fagan W, Schade J, Hood J, Sterner RW (2003). Growth rate-stoichiometry couplings in diverse biota. Ecology Letters, 6, 936-943.

Fanin N, Fromin N, Buatois B, Hättenschwiler S (2013). An experimental test of the hypothesis of non-homeostatic consumer stoichiometry in a plant litter-microbe system. Ecology Letters, 16, 764-772.

Fierer N, Bradford MA, Jackson RB (2007). Toward an ecological classification of soil bacteria. Ecology, 88, 1354-1364.

Fisk M, Santangelo S, Minick K (2015). Carbon mineralization is promoted by phosphorus and reduced by nitrogen addition in the organic horizon of northern hardwood forests. Soil Biology \& Biochemistry, 81, 212-218.

Frost PC, Benstead JP, Cross WF, Hillebrand H, Larson JH, Xenopoulos MA, Yoshida T (2006). Threshold elemental ratios of carbon and phosphorus in aquatic consumers. Ecology Letters, 9, 774-779.

Goberna M, Sánchez J, Pascual JA, García C (2006). Surface and subsurface organic carbon, microbial biomass and activity in a forest soil sequence. Soil Biology \& Biochemistry, 38, 2233-2243.

Han WX, Tang, LY, Chen YH, Fang JY (2013). Relationship between the relative limitation and resorption efficiency of nitrogen vs phosphorus in woody plants. PLOS ONE, 8, e83366

Harmon ME, Franklin JF, Swanson FJ, Sollins P, Gregory SV, Lattin JD, Anderson NH, Cline SP, Aumen NG, Sedell JR, Lienkaemper GW, Cromack KJ, Cummins KW (1986). Ecology of coarse woody debris in temperate ecosystems. Advances in Ecological Research, 15, 133-302.

Hartman WH, Richardson CJ (2013). Differential nutrient limitation of soil microbial biomass and metabolic quotients $\left(q \mathrm{CO}_{2}\right)$ : Is there a biological stoichiometry of soil microbes. PloS ONE, 8, e57127.

Hessen DO, Elser JJ, Sterner RW, Urabe J (2013). Ecological stoichiometry: An elementary approach using basic principles. Limnology \& Oceanography, 58, 2219-2236.

Högberg P, Nordgren A, Buchmann N, Taylor AF, Ekblad A, Högberg MN, Read DJ (2001). Large-scale forest girdling shows that current photosynthesis drives soil respiration.
Nature, 411, 789-792.

Ihsam H, Haselwandter K (1989). Metabolic quotient of the soil microflora in relation to plant succession. Oecologia, 79, 174-178.

Ilg K, Wellbrock N, Lux W (2009). Phosphorus supply and cycling at long-term forest monitoring sites in Germany. European Journal of Forest Research, 128, 483-492.

Jansson JK, Prosser JI (2013). Microbiology: The life beneath our feet. Nature, 494, 40-41.

Jetten MS (2008). The microbial nitrogen cycle. Environmental Microbiology, 10, 2903-2909.

Kaiser C, Franklin O, Dieckmann U, Richter A (2014). Microbial community dynamics alleviate stoichiometric constraints during litter decay. Ecology letters, 17, 680-690.

Lee SH, Jang I, Chae N, Choi T, Kang H (2013). Organic layer serves as a hotspot of microbial activity and abundance in Arctic tundra soils. Microbial ecology, 65, 405-414.

Leff JW, Jones SE, Prober SM, Barberán A, Borer ET, Firn JL, Fierer N (2015). Consistent responses of soil microbial communities to elevated nutrient inputs in grasslands across the globe. Proceedings of the National Academy of Sciences of the United States of America, 112, 1096710972.

Li P, Han WX, Yang YH, Fang JY (2014). Global patterns of soil microbial nitrogen and phosphorus stoichiometry in forest ecosystems. Global Ecology and Biogeography, 23, 979-987.

Li R, Chang RY (2015). Effects of external nitrogen additions on soil organic carbon dynamics and the mechanism. Chinese Journal of Plant Ecology, 39, 1012-1020. (in Chinese with English abstract) [李嵘, 常瑞英 (2015). 土壤有机 碳对外源氮添加的响应及其机制. 植物生态学报, 39, 1012-1020.]

Li Y, Wu JS, Liu SL, Shen JL, Huang DY, Su YR, Wei WW, Syers JK (2012). Is the C:N:P stoichiometry in soil and soil microbial biomass related to the landscape and land use in southern subtropical China? Global Biogeochemical Cycles, 26, GB4002. doi: 10.1029/2012GB004399.

Lovelock CE, Feller IC, Ball MC, Ellis J, Sorrell B (2007). Testing the growth rate $v s$. geochemical hypothesis for latitudinal variation in plant nutrients. Ecology Letters, 10, 1154-1163.

Lu XQ, Toda H, Ding FJ, Fang SZ, Yang WX, Xu HG (2014). Effect of vegetation types on chemical and biological properties of soils of karst ecosystems. European Journal of Soil Biology, 61, 49-57.

Makino W, Cotner JB, Sterner RW, Elser JJ (2003). Are bacteria more like plants or animals? Growth rate and resource dependence of bacterial C: N: P stoichiometry. Functional Ecology, 17, 121-130.

Manzoni S, Taylor P, Richter A, Porporato A, Ågren GI (2012). Environmental and stoichiometric controls on microbial carbon-use efficiency in soils. New Phytologist, 196, 
79-91.

Manzoni S, Trofymow JA, Jackson RB, Porporato A (2010). Stoichiometric controls on carbon, nitrogen, and phosphorus dynamics in decomposing litter. Ecological Monographs, 80, 89-106.

Marklein AR, Houlton BZ (2012). Nitrogen inputs accelerate phosphorus cycling rates across a wide variety of terrestrial ecosystems. New Phytologist, 193, 696-704.

Martin KJ, Picioreanu C, Nerenberg R (2015). Assessing microbial competition in a hydrogen-based membrane biofilm reactor (MBfR) using multidimensional modeling. Biotechnology \& Bioengineering, 112, 1843-1853.

Martinelli LA, Almeida S, Brown IF, Moreira MZ, Victoria RL, Filoso S, Ferreira CAC, Thomas WW (2000). Variation in nutrient distribution and potential nutrient losses by selective logging in a humid tropical forest of Rondonia, Brazil. Biotropica, 32, 597-613.

McGroddy ME, Daufresne T, Hedin LO (2004). Scaling of $\mathrm{C}: \mathrm{N}: \mathrm{P}$ stoichiometry in forests worldwide: Implications of terrestrial redfield-type ratios. Ecology, 85, 2390-2401.

Moore J, Macalady JL, Schulz MS, White AF, Brantley SL (2010). Shifting microbial community structure across a marine terrace grassland chronosequence, Santa Cruz, California. Soil Biology \& Biochemistry, 42, 21-31.

Moore JA, Jiang J, Patterson CM, Mayes MA, Wang G, Classen AT (2015). Interactions among roots mycorrhizas and free-living microbial communities differentially impact soil carbon processes. Journal of Ecology, 103, 14421453.

Moorhead DL, Sinsabaugh RL (2006). A theoretical model of litter decay and microbial interaction. Ecological Monographs, 76, 151-174.

Mooshammer M, Wanek W, Schnecker J, Wild B, Leitner S, Hofhansl F, Blöchl A, Hämmerle I, Frank AH, Keiblinger KM, Zechmeister-Boltenstern S, Richter A (2012). Stoichiometric controls of nitrogen and phosphorus cycling in decomposing beech leaf litter. Ecology, 93, 770-782.

Mooshammer M, Wanek W, Zechmeister-Boltenstern S, Richter A (2014). Stoichiometric imbalances between terrestrial decomposer communities and their resources: Mechanisms and implications of microbial adaptations to their resources. Frontiers in Microbiology, 5, 22.

Odum EP (1969). The strategy of ecosystem development. Science, 164, 262-270

Prosser JI, Bohannan BJ, Curtis TP, Ellis RJ, Firestone MK, Freckleton RP, Young JPW (2007). The role of ecological theory in microbial ecology. Nature Reviews Microbiology, 5, 384-392.

Raiesi F, Beheshti A (2015). Microbiological indicators of soil quality and degradation following conversion of native forests to continuous croplands. Ecological Indicators, 50, 173-185.
Redfield A (1958). The biological control of chemical factors in the environment. American Scientist, 46, 205-221.

Reed SC, Townsend AR, Davidson EA, Cleveland CC (2012). Stoichiometric patterns in foliar nutrient resorption across multiple scales. New Phytologist, 196, 173-180.

Richardson AE, Simpson RJ (2011). Soil microorganisms mediating phosphorus availability update on microbial phosphorus. Plant Physiology, 156, 989-996.

Rick WY, Thomas SM (2001). Microbial nitrogen cycles: Physiology genomics and applications. Current Opinion in Microbiology, 4, 307-312.

Sardans J, Peñuelas J (2012). The role of plants in the effects of global change on nutrient availability and stoichiometry in the plant-soil system. Plant Physiology, 160, 1741-1761.

Sardans J, Rivas-Ubach A, Peñuelas J (2012). The elemental stoichiometry of aquatic and terrestrial ecosystems and its relationships with organismic lifestyle and ecosystem structure and function: A review and perspectives. Biogeochemistry, 111, 1-39.

Saviozzi A, Levi-Minzi R, Cardelli R, Riffaldi R (2001). A comparison of soil quality in adjacent cultivated, forest and native grassland soils. Plant \& Soil, 233, 251-259.

Schimel JP, Weintraub MN (2003). The implications of exoenzyme activity on microbial carbon and nitrogen limitation in soil: A theoretical model. Soil Biology \& Biochemistry, 35, 549-563.

Schneider T, Keiblinger KM, Schmid E, Sterflinger-Gleixner K, Ellersdorfer G, Roschitzki B, Richter A, Eberl L, Zechmeister-Boltenstern S, Riedel K (2012). Who is who in litter decomposition? Metaproteomics reveals major microbial players and their biogeochemical functions. ISME Journal, 6, 1749-1762.

Seeling B, Zasoski RJ (1993). Microbial effects in maintaining organic and inorganic solution phosphorus concentrations in a grassland topsoil. Plant \& Soil, 148, 277-284.

Sinsabaugh R, Follstad Shah JJ (2012). Ecoenzymatic stoichiometry and ecological theory. Annual Review of Ecology, Evolution, \& Systematics, 43, 313-343.

Sinsabaugh RL, Follstad Shah JJ (2011). Ecoenzymatic stoichiometry of recalcitrant organic matter decomposition: The growth rate hypothesis in reverse. Biogeochemistry, 102, 31-43.

Sinsabaugh RL, Hill BH, Follstad Shah JJ (2009). Ecoenzymatic stoichiometry of microbial organic nutrient acquisition in soil and sediment. Nature, 462, 795-798.

Sinsabaugh RL, Lauber CL, Weintraub MN, Ahmed B, Allison SD, Crenshaw C, Contosta AR, Cusack D, Frey S, Gallo ME (2008). Stoichiometry of soil enzyme activity at global scale. Ecology Letters, 11, 1252-1264.

Sinsabaugh RL, Manzoni S, Moorhead DL, Richter A (2013). Carbon use efficiency of microbial communities: Stoichiometry, methodology and modelling. Ecology Letters, 16, 930-939. 
Spohn M (2015). Microbial respiration per unit microbial biomass depends on litter layer carbon-to-nitrogen ratio. Biogeosciences, 12, 817-823.

Spohn M, Chodak M (2015). Microbial respiration per unit biomass increases with carbon-to-nutrient ratios in forest soils. Soil Biology \& Biochemistry, 81, 128-133.

Steinauer K, Tilman D, Wragg PD, Cesarz S, Cowles JM, Pritsch K, Reich PB, Weisser WW, Eisenhauer N (2015). Plant diversity effects on soil microbial functions and enzymes are stronger than warming in a grassland experiment. Ecology, 96, 99-112.

Sterner RW, Elser JJ (2002). Ecological Stoichiometry: The Biology of Elements from Molecules to the Biosphere. Princeton University Press, Princeton.

Strickland MS, Osburn E, Lauber C, Fierer N, Bradford MA (2009). Litter quality is in the eye of the beholder: Initial decomposition rates as a function of inoculum characteristics. Functional Ecology, 23, 627-636.

Strickland MS, Rousk J (2010). Considering fungal: Bacterial dominance in soils-methods, controls, and ecosystem implications. Soil Biology \& Biochemistry, 42, 1385-1395.

Susyan EA, Wirth S, Ananyeva, ND, Stolnikova EV (2011). Forest succession on abandoned arable soils in European Russia-Impacts on microbial biomass, fungal-bacterial ratio, and basal $\mathrm{CO}_{2}$ respiration activity. European Journal of Soil Biology, 47, 169-174.

Tian HQ, Chen GS, Zhang C, Melillo JM, Hall CAS (2010). Pattern and variation of C:N:P ratios in China's soils: A synthesis of observational data. Biogeochemistry, 98, 139-151.

van der Heijden MG, Bardgett RD, van Straalen NM (2008). The unseen majority: Soil microbes as drivers of plant diversity and productivity in terrestrial ecosystems. Ecology letters, 11, 296-310.

Vergutz L, Manzoni S, Porporato A, Novais RF, Jackson RB (2012). Global resorption efficiencies and concentrations of carbon and nutrients in leaves of terrestrial plants. Ecological Monographs, 82, 205-220.

Vitousek PM, Howarth RW (1991). Nitrogen limitation on land and in the sea: How can it occur? Biogeochemistry, 13, 87-115.

Vitousek PM, Porder S, Houlton BZ, Chadwick OA (2010). Terrestrial phosphorus limitation: Mechanisms implications and nitrogen-phosphorus interactions. Ecological Applications, 20, 5-15.

Wang CK, Han Y, Chen JQ, Wang XC, Zhang QZ, Bond-Lamberty B (2013). Seasonality of soil $\mathrm{CO}_{2}$ efflux in a temperate forest: Biophysical effects of snowpack and spring freeze-thaw cycles. Agricultural \& forest meteorology, 177, 83-92.

Wang CK, Yang JY (2007). Rhizospheric and heterotrophic components of soil respiration in six Chinese temperate forests. Global Change Biology, 13, 123-131.
Wang CK, Yang JY, Zhang QZ (2006). Soil respiration in six temperate forests in China. Global Change Biology, 12, 2103-2114.

Wang CS, Wang SP (2015). A review of research on responses of leaf traits to climate change. Chinese Journal of Plant Ecology, 39, 206-216. (in Chinese with English abstract) [王常顺, 汪诗平 (2015). 植物叶片性状对气候变化的 响应研究进展. 植物生态学报, 39, 206-216.]

Wang XC, Wang CK (2015). Fundamental concepts and field measurement methods of carbon cycling in forest ecosystems: a review. Acta Ecologica Sinica, 35, 4241-4256. (in Chinese with English abstract) [王兴昌, 王传宽 (2015). 森林生态系统碳循环的基本概念和野外测定方法评述. 生态学报, 35, 4241-4256.]

Wardle DA, Ghani A (1995). A critique of the microbial metabolic quotient $\left(q_{\mathrm{CO}_{2}}\right)$ as a bioindicator of disturbance and ecosystem development. Soil Biology \& Biochemistry, 27, 1601-1610.

Waring BG, Weintraub SR, Sinsabaugh RL (2014). Ecoenzymatic stoichiometry of microbial nutrient acquisition in tropical soils. Biogeochemistry, 117, 101-113.

Weedon JT, Cornwell WK, Cornelissen JHC, Zanne AE, Wirth C, Coomes DA (2009). Global meta-analysis of wood decomposition rates: A role for trait variation among tree species? Ecology Letters, 12, 45-56.

Xu XF, Schimel JP, Thornton PE, Song X, Yuan FM, Goswami S (2014). Substrate and environmental controls on microbial assimilation of soil organic carbon: A framework for Earth system models. Ecology Letters, 17, 547-555.

Xu XF, Thornton PE, Post WM (2013). A global analysis of soil microbial biomass carbon, nitrogen and phosphorus in terrestrial ecosystems. Global Ecology \& Biogeography, 22, 737-749.

Yang X, Huang Z, Zhang K, Cornelissen JH (2015). C:N:P stoichiometry of Artemisia species and close relatives across northern China: Unravelling effects of climate, soil and taxonomy. Journal of Ecology, 103, 1020-1031.

Yang YH, Luo YQ (2011). Carbon:nitrogen stoichiometry in forest ecosystems during stand development. Global Ecology \& Biogeography, 20, 354-361.

Yang YH, Luo YQ, Finzi AC (2011). Carbon and nitrogen dynamics during forest stand development: A global synthesis. New Phytologist, 190, 977-989.

Yuan ZY, Chen HY (2009). Global-scale patterns of nutrient resorption associated with latitude, temperature and precipitation. Global Ecology \& Biogeography, 18, 11-18.

Yuan ZY, Chen HY, Reich PB (2011). Global-scale latitudinal patterns of plant fine-root nitrogen and phosphorus. Nature Communications, 2, 344.

Zechmeister-Boltenstern S, Keiblinger KM, Mooshammer M, Peñuelas J, Richter A, Sardans J, Wanek W (2015). The application of ecological stoichiometry to plant-microbial- 
soil organic matter transformations. Ecological Monographs, 85, 133-155.

Zelezniak A, Andrejev S, Ponomarova O, Mende DR, Bork P, Patil KR (2015). Metabolic dependencies drive species co-occurrence in diverse microbial communities. Proceedings of the National Academy of Sciences of the United States of America, 112, 6449-6454.

Zhang QZ, Wang CK (2010). Carbon density and distribution of six Chinese temperate forests. Science China (Life Science), 53, 831-840.

Zhou ZH, Wang CK (2015). Reviews and syntheses: Soil resources and climate jointly drive variations in microbial biomass carbon and nitrogen in China's forest ecosystems. Biogeosciences, 12, 6751-6760.

Zhou ZH, Wang CK, Zhang QZ (2015). The effect of land use change on soil carbon, nitrogen, and phosphorus contents and their stoichiometry in temperate sapling stands in northeastern China. Acta Ecologica Sinica, 35, 6694- 6702. (in Chinese with English abstract)[周正虎, 王传宽, 张全 智 (2015). 土地利用变化对东北温带幼龄林土壤碳氮 磷含量及其化学计量特征的影响. 生态学报, 35, 6694-6702.]

责任编委: 韩文轩 责任编辑: 李 敏

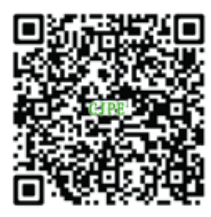

植物生态学报官网

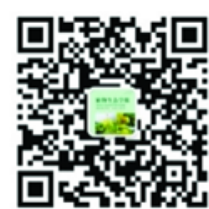

微信订阅号

期刊及学科

相关信息发布

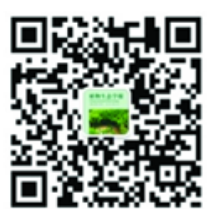

微信服务号

稿件状态查询 全文检索汶览 